\title{
Testing the Sunyaev-Zeldovich-based tomographic approach to the thermal history of the Universe with pressure-density cross correlations: Insights from the Magneticum simulation
}

\author{
Sam Young $\odot^{*}$ and Eiichiro Komatsu $\odot^{\dagger}$ \\ Max Planck Institute for Astrophysics, Karl-Schwarzschild-Str. 1, 85748 Garching, Germany \\ Klaus Dolag \\ University Observatory Munich, Scheinerstr. 1, 81679 Munich, Germany \\ and Max Planck Institute for Astrophysics, Karl-Schwarzschild-Str. 1, 85748 Garching, Germany
}

(Received 7 June 2021; accepted 16 September 2021; published 22 October 2021)

\begin{abstract}
The thermal Sunyaev-Zeldovich effect contains information about the thermal history of the Universe, which is observable in maps of the Compton $y$ parameter; however, it does not contain information about the redshift of the sources. Recent papers have utilized a tomographic approach, by cross correlating the Compton $y$ map with the locations of galaxies with known redshift in order to deproject the signal along the line of sight. In this paper, we test the validity and accuracy of this tomographic approach to probe the thermal history of the Universe. We use the state-of-the-art, cosmological, and hydrodynamical simulation, Magneticum, for which the thermal history of the Universe is a known quantity. The key ingredient is the Compton- $y$-weighted halo bias, $b_{y}$, which is computed from the halo model. We find that, at redshifts currently available, the method reproduces the correct mean thermal pressure (or the density-weighted mean temperature) with high accuracy, validating and confirming the results of previous papers. At higher redshifts $(z \gtrsim 2)$, there is significant disagreement between $b_{y}$ from the halo model and the simulation.
\end{abstract}

DOI: $10.1103 /$ PhysRevD.104.083538

\section{INTRODUCTION}

As cosmological structures form, the gravitational potential wells seeded by primordial density fluctuations become deeper [1]. As this process occurs, gravitational potential energy is converted into kinetic energy in an expanded Universe, following the Layzer-Irvine equation [2-4]. A part of the kinetic energy is converted into thermal energy via the process of shock heating in the large-scale structure of the Universe [5-7], while the rest remains in the form of bulk or turbulent motion until it decays and thermalizes [8,9]. Therefore, measurements of the mean thermal energy of the cosmic gas content at different redshifts can be used to probe the growth of structure, as recently demonstrated in Refs. [10,11].

\footnotetext{
syoung@mpa-garching.mpg.de

${ }^{\dagger}$ Also at Kavli Institute for the Physics and Mathematics of the Universe (Kavli IPMU, WPI), Todai Institutes for Advanced Study, The University of Tokyo, Kashiwa 277-8583, Japan.

Published by the American Physical Society under the terms of the Creative Commons Attribution 4.0 International license. Further distribution of this work must maintain attribution to the author(s) and the published article's title, journal citation, and DOI. Open access publication funded by the Max Planck Society.
}

To this end, the thermal Sunyaev-Zeldovich (SZ) effect $[12,13]$ can be used to probe the baryons in the Universe [14-16]. As photons in the cosmic microwave background (CMB) are inverse Compton scattered off of the free electrons in the ionized gas, they leave an imprint in the form of a spectral distortion in the CMB. The amplitude of the SZ effect depends on the electron pressure integrated along the line of sight, which is parametrized by the Compton $y$ parameter $[12,13]$,

$$
y(\hat{\phi})=\frac{\sigma_{\mathrm{T}}}{m_{\mathrm{e}} c^{2}} \int \frac{\mathrm{d} \chi}{1+z} P_{\mathrm{e}}(\chi \hat{\phi}),
$$

where $\sigma_{\mathrm{T}}$ is the Thomson scattering cross section, $m_{\mathrm{e}}$ is the electron mass, $c$ is the speed of light, $\chi=\chi(z)$ is the comoving radial distance out to a given redshift $z$, and $P_{\mathrm{e}}$ is the electron pressure. The integral runs from zero up to the surface of last scattering, $z \simeq 1090$. The electron pressure $P_{\mathrm{e}}$ is related to the electron temperature $T_{\mathrm{e}}$ as $P_{\mathrm{e}}=n_{\mathrm{e}} k_{\mathrm{B}} T_{\mathrm{e}}$, where $n_{\mathrm{e}}$ and $k_{\mathrm{B}}$ are the (proper) electron number density and the Boltzmann constant, respectively.

The SZ effect, which is observable in maps of the Compton $y$ parameter, contains information about $P_{\mathrm{e}}$ but does not contain information about $z$ of the sources. To probe the growth history of structure using the SZ effect, we therefore require an external source of information 
about $z$ [17,18]. As the SZ signal is dominated by massive structure, the signal can be deprojected along the line of sight using clustering-based redshift inference [19-21]. Following this method, an external sample of reference sources with known $z$ is taken, and cross correlated with the Compton $y$ parameter as a function of $z$. This allows correlated intensities to be extracted tomographically [22-24], as has been studied in recent papers for the SZ-galaxy cross correlation with spectroscopic redshifts $[10,25,26]$. See Refs. [27-29] for the cross correlation with photometric redshifts.

The halo bias-weighted mean electron pressure, $\left\langle b P_{\mathrm{e}}\right\rangle$, is the direct observable of the SZ-galaxy cross-correlation function on large scales [25]. Here, $\langle\ldots\rangle$ denotes an ensemble average. To infer the mean electron pressure, $\left\langle P_{\mathrm{e}}\right\rangle$, we need to know the Compton $y$-weighted halo bias, $b_{y} \equiv\left\langle b P_{\mathrm{e}}\right\rangle /\left\langle P_{\mathrm{e}}\right\rangle$. In Refs. [10,11], the halo model developed in Refs. [26,30-33] was used to calculate $b_{y}$ and infer $\left\langle P_{\mathrm{e}}\right\rangle$ from the measured $\left\langle b P_{\mathrm{e}}\right\rangle$.

How accurate is this approach? It is the aim of this paper to test the validity of the tomographic approach to the mean thermal history of the Universe in Refs. [10,11]. Specifically, we test this approach against the Magneticum simulation [34]. We use cross correlations of the density and pressure to calculate the density-weighted mean temperature of baryonic gas, which is a known quantity in the simulation.

The rest of this paper is organized as follows. In Sec. II, we describe the Magneticum simulation. We then present the comparison of the simulation and observations for $\left\langle b P_{\mathrm{e}}\right\rangle$ in Sec. III and the density-weighted mean electron temperature $\bar{T}_{\mathrm{e}}$ in Sec. V, while we compare the halo model calculation and the simulation results for $b_{y}$ in Sec. IV. We conclude in Sec. VI.

\section{THE MAGNETICUM SIMULATION}

The Magneticum simulation is a set of state-of-the-art, cosmological, and hydrodynamical simulations of different cosmological volumes with different resolutions, performed with an improved version of the smoothed-particle hydrodynamics (SPH) code GADGET3 [35,36]. They follow a standard $\Lambda$ cold dark matter (CDM) cosmology with parameters close to the best-fitting values of the WMAP 7-year results [37] for a flat $\Lambda \mathrm{CDM}$ cosmology with the total matter density $\Omega_{\mathrm{m}}=0.272$ (16.8\% baryons), the cosmological constant $\Omega_{\Lambda}=0.728$, the Hubble constant $H_{0}=70.4 \mathrm{~km} \mathrm{~s}^{-1} \mathrm{Mpc}^{-1}(h=0.704)$, the index of the primordial power spectrum $n_{\mathrm{s}}=0.963$, and the overall normalization of the power spectrum $\sigma_{8}=0.809$.

Here, we describe the simulations briefly. For more detailed descriptions, we refer to previous work using these simulations [34,38-42]. The simulations follow a wide range of physical processes (see Refs. $[43,44]$ for details), which are important for studying the formation of active galactic nuclei (AGN), galaxies, and galaxy groups and clusters. The simulation set covers a huge dynamical range that follows the same underlying treatment of the physical processes controlling galaxy formation, thereby allowing them to reproduce the properties of the large-scale, intergalactic, and intracluster mediums [34,39,45], as well as the detailed properties of galaxies, including morphological classifications and internal properties $[44,46,47]$. This also includes the distribution of different metal species within galaxies and galaxy clusters [48] and the properties of the AGN population $[43,49]$. Especially, the simulations well reproduce the observed pressure profiles of galaxy clusters $[39,50]$ and $\mathrm{x}$-ray scaling relations [51].

Here, we focus on the largest box ("Box0"), which follows the evolution of $2 \times 4536^{3}$ particles in a large box of the comoving volume $\left(2688 \mathrm{~h}^{-1} \mathrm{Mpc}\right)^{3}$ [38,40,52], making it the largest cosmological and hydrodynamical simulation to reach $z=0$ performed to date.

\section{THE HALO BIAS-WEIGHTED MEAN ELECTRON PRESSURE $\left\langle b_{\mathrm{e}}\right\rangle$}

The tomographic technique provides direct constraints on the halo bias-weighted mean electron pressure, $\left\langle b P_{\mathrm{e}}\right\rangle$ [25]. This can be rewritten using the large-scale, $y$ weighted halo clustering bias $b_{y}$ and the mean electron pressure $\left\langle P_{\mathrm{e}}\right\rangle$ as $\left\langle b P_{\mathrm{e}}\right\rangle=b_{y}\left\langle P_{\mathrm{e}}\right\rangle$ [10]. By making use of the Magneticum simulation, knowledge of the density and pressure is readily available for different $z$, and instead of using the clustering redshift technique, we can perform an equivalent measurement using a cross correlation of the density $\rho$ and thermal gas pressure $P_{\mathrm{th}}$ :

$$
\frac{\left\langle\delta_{\rho} P_{\mathrm{th}}\right\rangle(k)}{\left\langle\delta_{\rho} \delta_{\rho}\right\rangle(k)} \rightarrow\left\langle b P_{\mathrm{th}}\right\rangle=b_{y}\left\langle P_{\mathrm{th}}\right\rangle,
$$

where $\delta_{\rho} \equiv(\rho-\bar{\rho}) / \bar{\rho}$ and the bar denotes the mean value in the simulation. Assuming the gas is fully ionized, the electron pressure $P_{\mathrm{e}}$ is related to the total thermal gas pressure $P_{\text {th }}$ as $P_{\text {th }}=(8-5 Y) /(4-2 Y) P_{\mathrm{e}}$, where $Y=$ 0.24 is the primordial helium mass fraction (we assume a primordial mixture of elements and full ionization). Here, $\left\langle\delta_{\rho} P_{\text {th }}\right\rangle(k)$ and $\left\langle\delta_{\rho} \delta_{\rho}\right\rangle(k)$ denote the density-pressure cross power spectrum and the density power spectrum, respectively, and $k$ is the wave number. The arrow indicates the large-scale limit, $k \rightarrow 0$.

Why do we call $\left\langle b P_{\text {th }}\right\rangle$ the "halo-bias weighted" quantity, when we cross correlate the pressure and density fields with no explicit reference to collapsed structures such as halos? The reason is that the thermal pressure is dominated by halos [6,31]; specifically, the density-pressure cross correlation is dominated by galaxy groups and clusters $[10,11]$. Therefore, our estimator using the density fields yields the halo-bias weighted mean pressure.

In order to obtain $\left\langle\delta_{\rho} P_{\text {th }}\right\rangle(k)$ and $\left\langle\delta_{\rho} \delta_{\rho}\right\rangle(k)$, particles in the Magneticum simulation are assigned to the nearest point 


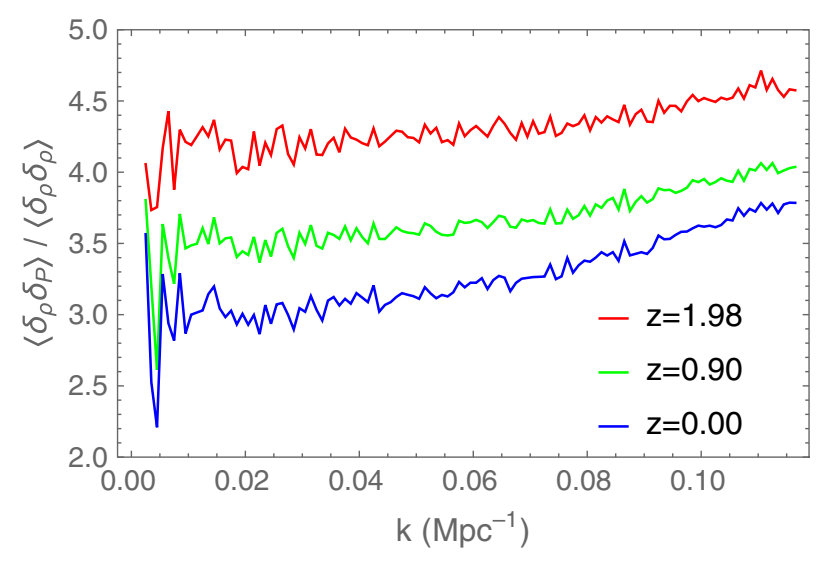

FIG. 1. The ratio of the density-pressure cross power spectrum and the density power spectrum, $\left\langle\delta_{\rho} \delta_{P}\right\rangle(k) /\left\langle\delta_{\rho} \delta_{\rho}\right\rangle(k)$ with $\delta_{P} \equiv\left(P_{\text {th }}-\bar{P}_{\text {th }}\right) / \bar{P}_{\text {th }}$, as measured in the Magneticum simulation. We show the results for three different redshifts: $z=1.98$, 0.90 , and 0.00 (from top to bottom). At large scales, the value approaches a constant value, $b_{y}$, to within scatter due to the finite box size.

in a $100^{3}$ grid spanning a simulation's box. The coarse grain of the grid is acceptable since we are only interested in the large-scale limit of the cross correlation. The nbodykit package [53] is used to calculate the power spectra and cross correlation of the resulting maps.

In Fig. 1, we show the ratio $\left\langle\delta_{\rho} \delta_{P}\right\rangle(k) /\left\langle\delta_{\rho} \delta_{\rho}\right\rangle(k)$ as a function of $k$, where $\delta_{P} \equiv\left(P_{\text {th }}-\bar{P}_{\text {th }}\right) / \bar{P}_{\text {th }}$. This quantity is equal to $b_{y}$ in the large-scale limit. We obtain the largescale value by averaging the correlation functions over $k<0.03 \mathrm{Mpc}^{-1}$. This reduces the scatter in the correlation functions at small $k$ due to the finite box size. We have checked for convergence with respect to our choice of $k<0.03 \mathrm{Mpc}^{-1}$. The standard deviation in the correlation functions is used to derive error bars on the quantities derived using this method. We also checked that fitting the correlation functions to various forms has only a small effect on the calculated values for $b_{y}$ of approximately $2 \%$, which is subdominant to the uncertainty due to cosmic variance.

We estimate the uncertainty due to cosmic variance by dividing the simulation box into 64 subregions and calculating the variance of $\left\langle b P_{\mathrm{e}}\right\rangle$ within those regions. In Fig. 2, we compare the values of $\left\langle b P_{\mathrm{e}}\right\rangle$ from the Magneticum simulation and the observations [10]. They are in excellent agreement. To quantify how well the Magneticum values fit the data, we consider an overall rescaling of the electron pressure $\left\langle b P_{\mathrm{e}}\right\rangle \rightarrow C\left\langle b P_{\mathrm{e}}\right\rangle$, and consider if this can give a better fit to the data as presented in [10]. A $\chi^{2}$ analysis, comparing the Magneticum values with the data up to $z=$ 0.98 (above this value only upper bounds are available), gives a value $C=0.936 \pm 0.095$. This confirms that the measurements from the Magneticum simulation are in agreement with observations-with an uncertainty at the $10 \%$ level.

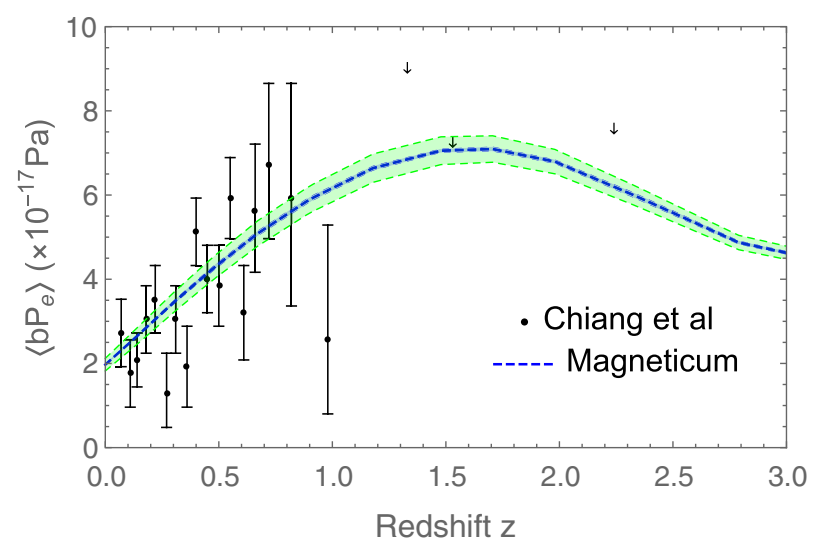

FIG. 2. The bias-weighted mean electron pressure $\left\langle b P_{\mathrm{e}}\right\rangle$ is shown as a function of $z$. The points are taken from the observed data given in Ref. [10], and the blue dashed line shows the value from the Magneticum simulation. The (small) shaded blue region shows the $1 \sigma$ uncertainty in the Magneticum simulation due to the scatter in the correlation functions at small $k$, while the shaded green region shows the uncertainty due to cosmic variance.

\section{THE LARGE-SCALE, $y$-WEIGHTED HALO CLUSTERING BIAS $\boldsymbol{b}_{\boldsymbol{y}}$}

While $\left\langle b P_{\mathrm{e}}\right\rangle$ is the direct observable of the SZ-based tomography, there is a degeneracy between the redshift of the SZ clusters and their bias. We therefore need the knowledge of $b_{y}$ to obtain $\left\langle P_{\mathrm{e}}\right\rangle$ from the observed $\left\langle b P_{\mathrm{e}}\right\rangle$. In Refs. [10,11], the halo model developed in Refs. [26,30-33], which resulted in the pysz code [54], was used to calculate $b_{y}$. One of the key outcomes of this paper is to determine the accuracy of this approach-since the bias $b_{y}$ can be computed directly within the Magneticum simulation and compared to the halo model calculation.

The halo model calculation yields (see Appendix B of [10] for details)

$$
b_{y}(z)=\frac{\int d M \frac{d n}{d M} M^{5 / 3+\alpha_{p}} b_{h}(M, z)}{\int d M \frac{d n}{d M} M^{5 / 3+\alpha_{p}}},
$$

where $d n / d M$ and $b_{h}$ are the mass function and linear clustering bias of dark matter halos, respectively $[55,56]$. The physics is simple: the total pressure of gas in a halo $\int d V P_{\mathrm{th}}(M)$ is proportional to the halo mass $M$ times the virial temperature, the latter of which is proportional to $M^{2 / 3}$; thus, the virial relation gives $\int d V P_{\text {th }} \propto M^{5 / 3}$. The X-ray observation of galaxy clusters shows a small empirical correction to this relation, $M^{5 / 3+\alpha_{p}}$ with $\alpha_{p}=0.12$ [57]. We use the pysz code to calculate $b_{y}$ with the following parameters: $h=0.704, \Omega_{\mathrm{b}} h^{2}=0.02265, \Omega_{\mathrm{c}} h^{2}=0.11216$, $A_{\mathrm{s}}=2.42 \times 10^{-9}$, and $n_{\mathrm{s}}=0.963$. The neutrino mass $m_{\nu}$ is set to zero because the Magneticum simulation does not include massive neutrinos. 


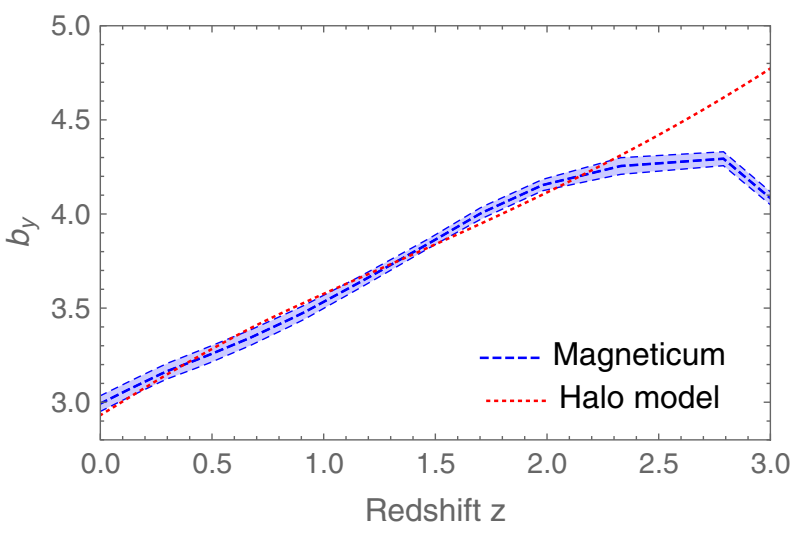

FIG. 3. The $y$-weighted halo bias $b_{y}$ is shown as a function of $z$. The red, dotted line is the prediction from the halo model, while the blue line is that derived from the Magneticum simulation. The shaded blue region represents the $1 \sigma$ uncertainty in $b_{y}$ due to scatter in the large-scale correlation functions. We find excellent agreement between the two until $z \gtrsim 2$.

In Fig. 3, we show the comparison of $b_{y}$ calculated with the halo model and from Magneticum. We find that for low $z$, there is excellent agreement between the two calculations-well within the uncertainties. However, at large $z \gtrsim 2$, there is significant disagreement, with $b_{y}$ measured in Magneticum being significantly lower than predicted by the halo model. This suggests that the measurements made in Ref. [10] of the mean electron temperature (see Sec. V) are reliable up to a redshift $z \lesssim 2$, but implies uncertainty in the upper bounds which were derived at $z \gtrsim 2$.

The reason for this disagreement is not clear, but it is plausible that the assumption (made for the halo model) that pressure is dominated by the virialized structures and the contribution from supernova and AGN feedback are subdominant compared to the thermal pressure of virialized gas may be violated at such a high $z$. There may be an additional term arising from a response of internal properties of galaxy groups and clusters to the large-scale overdensity $[58,59]$, which we do not include in Eq. (3). As Eq. (3) agrees with $b_{y}$ from the Magneticum simulation, which should include all the relevant effects, precisely at $z \lesssim 2$, these contributions are small in this redshift range. Further analysis of this disagreement at $z \gtrsim 2$ is left for future work.

To quantify how well the Magneticum values fit the halo model prediction, we again consider an overall rescaling factor to the halo bias calculated with the halo model, $b_{y} \rightarrow A b_{y}$, and consider whether this can provide a better fit to the simulation. Fitting only to values from $z<2.5$, and performing a $\chi^{2}$ analysis, the value $A=1.0027 \pm 0.0043$ is given, indicating that the halo model prediction is able to predict the values from Magneticum to better than $1 \%$ accuracy.

\section{THE DENSITY-WEIGHTED MEAN ELECTRON TEMPERATURE $\bar{T}_{\mathrm{e}}$}

The mean electron pressure $\left\langle P_{\mathrm{e}}\right\rangle$ is related to the densityweighted mean temperature of electrons in the Universe, defined as $\bar{T}_{\mathrm{e}} \equiv\left\langle n_{\mathrm{e}} T_{\mathrm{e}}\right\rangle /\left\langle n_{\mathrm{e}}\right\rangle \quad[5,6]$. Specifically, using $\left\langle b P_{\mathrm{e}}\right\rangle$ and $b_{y}$, we find

$$
\bar{T}_{\mathrm{e}}=\frac{2 m_{\mathrm{H}}}{\rho_{\mathrm{c}} \Omega_{\mathrm{b}} k_{\mathrm{B}}(2-Y)(1+z)^{3}} \frac{1}{b_{y}}\left\langle b P_{\mathrm{e}}\right\rangle,
$$

where $\rho_{\mathrm{c}}=3 H_{0}^{2} /(8 \pi G)$ is the critical density of the Universe at $z=0$ and $m_{\mathrm{H}}$ is the hydrogen mass.

The temperature data can also be read directly from the output files of the Magneticum simulation, and the uncertainty in the mean temperature (and pressure) due to cosmic variance is estimated by dividing a simulation's box into 64 subregions and calculating the variance of the mean temperature within those subregions. We assume throughout that the electron temperature and the total temperature are equal, although there is likely to be some difference between the two, as discussed in more detail in Refs. [60,61].

For a direct comparison to the measurements of Ref. [10], we here use the value of $b_{y}$ calculated using the halo model, rather than the measured value from the Magneticum simulation. In Fig. 4 , we compare $\bar{T}_{\mathrm{e}}$ from the data and the simulation as a function of $z$. As expected from the excellent agreement for $\left\langle b P_{\mathrm{e}}\right\rangle$ (Fig. 2) and $b_{y}$ (Fig. 3), we find excellent agreement for $\bar{T}_{\mathrm{e}}$. This completes the validation of the methodology for obtaining $\left\langle T_{\mathrm{e}}\right\rangle$, i.e., the thermal history of the Universe, developed in Refs. [10,11].

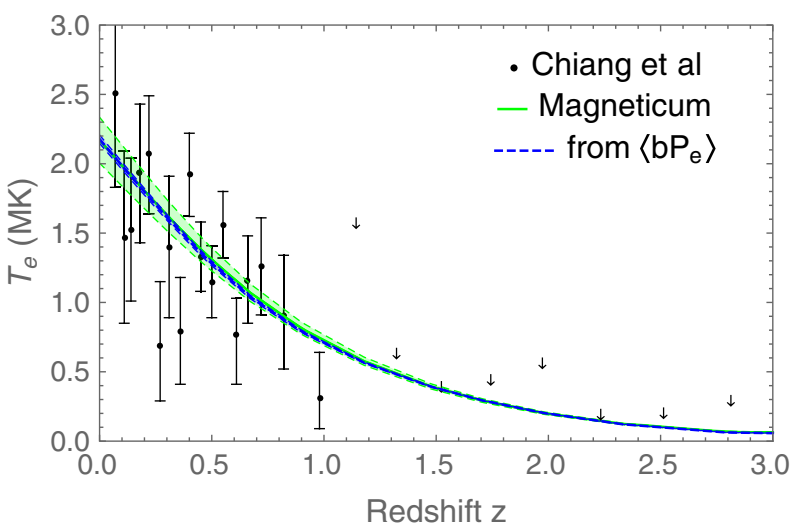

FIG. 4. The density-weighted mean electron temperature $\bar{T}_{\mathrm{e}}$ (in units of million $\mathrm{K}$ ) is shown as a function of $z$. The points are taken from the observed data given in Ref. [10]. The solid green line shows the temperature read directly from the Magneticum simulation, while the blue dashed line shows that calculated from the pressure data in the Magneticum simulation. The blue and green regions show the $1 \sigma$ confidence intervals for their respective data. 


\section{CONCLUSION}

This study aimed to answer two principle questions:

(1) The bias-weighted mean electron pressure $\left\langle b P_{\mathrm{e}}\right\rangle$ is observable from cosmological surveys. Does this quantity measured from the Magneticum simulation agree with the data given in Ref. [10]?

(2) In Ref. [10], the density-weighted mean electron temperature $\bar{T}_{\mathrm{e}}$ is derived by dividing $\left\langle b P_{\mathrm{e}}\right\rangle$ by $b_{y}$, calculated from the halo model. Is this method accurate?

The results presented here show that the answer to both questions is yes. The values of $\left\langle b P_{\mathrm{e}}\right\rangle$ (as well as $\bar{T}_{\mathrm{e}}$ ) from Magneticum agree well with the data-with the best-fitting value to rescale the overall pressure given by $C=0.936 \pm 0.095$, consistent with unity to within the uncertainty. Likewise, the halo model prediction for $b_{y}$ matches very well to that measured in Magneticum, with the best-fitting rescaling value given by $A=1.0027 \pm 0.0043$.

The results presented here confirm the validity of the tomographic method in order to determine the thermal history of gas in the Universe, although they also highlight a need for further study of the halo model calculation at high redshifts, $z \gtrsim 2$, which are yet to be probed by observations.

We also suggest that the measured mean temperature can be used as a simple "thermometer test" of the baryonic physics of simulations to confirm that they are capable of accurately reproducing observed values, which can be performed for a host of state-of-the-art cosmological and hydrodynamical simulations [62-67].

\section{ACKNOWLEDGMENTS}

E. K. thanks Y.-K. Chiang, R. Makiya, and B. Ménard for the collaboration on Refs. [10,11], which motivated this work. S. Y. thanks R. Makiya for his help with the pysz code [26,33], and was supported during the research by a Humboldt Research Fellowship. This work was supported in part by the Deutsche Forschungsgemeinschaft (DFG, German Research Foundation) under Germany's Excellence Strategy-EXC-2094-390783311. K. D. acknowledges support for the COMPLEX project from the European Research Council (ERC) under the European Union's Horizon 2020 research and innovation program through Grant Agreement No. ERC-2019-AdG 860744. The Kavli I.P. M. U. is supported by World Premier International Research Center Initiative (WPI), MEXT, Japan. The calculations were carried out at the Leibniz Supercomputer Center (LRZ) under the Project No. pr83li. We are especially grateful for the support by M. Petkova through the Computational Center for Particle and Astrophysics (C2PAP) and the support by N. Hammer at LRZ when carrying out the Box0 simulation with the Extreme Scale-Out Phase on the new SuperMUC Haswell extension system.
[1] P. J. E. Peebles, The Large-Scale Structure of the Universe (Princeton University Press, Princeton, NJ, 1980).

[2] D. Layzer, A preface to cosmogony. I. The energy equation and the virial theorem for cosmic distributions, Astrophys. J. 138, 174 (1963).

[3] W. M. Irvine, Local irregularities in a universe satisfying the cosmological principle, Ph.D. thesis, Harvard University, 1961.

[4] N. A. Dmitriev and Y.B. Zeldovich, The energy of accidental motions in the expanding Universe, Sov. J. Exp. Theor. Phys. 18, 793 (1964).

[5] R. Cen and J.P. Ostriker, Where are the baryons?, Astrophys. J. 514, 1 (1999).

[6] A. Refregier, E. Komatsu, D. N. Spergel, and U.-L. Pen, Power spectrum of the Sunyaev-Zel'dovich effect, Phys. Rev. D 61, 123001 (2000).

[7] F. Miniati, D. Ryu, H. Kang, T. W. Jones, R. Cen, and J.P. Ostriker, Properties of cosmic shock waves in large scale structure formation, Astrophys. J. 542, 608 (2000).
[8] X. Shi and E. Komatsu, Analytical model for non-thermal pressure in galaxy clusters, Mon. Not. R. Astron. Soc. 442, 521 (2014).

[9] X. Shi, E. Komatsu, K. Nelson, and D. Nagai, Analytical model for non-thermal pressure in galaxy clusters-II. Comparison with cosmological hydrodynamics simulation, Mon. Not. R. Astron. Soc. 448, 1020 (2015).

[10] Y.-K. Chiang, R. Makiya, B. Ménard, and E. Komatsu, The cosmic thermal history probed by Sunyaev-Zeldovich effect tomography, Astrophys. J. 902, 56 (2020).

[11] Y.-K. Chiang, R. Makiya, E. Komatsu, and B. Ménard, The thermal and gravitational energy densities in the large-scale structure of the Universe, Astrophys. J. 910, 32 (2021).

[12] Y. B. Zeldovich and R. A. Sunyaev, The interaction of matter and radiation in a hot-model Universe, Astrophys. Space Sci. 4, 301 (1969).

[13] R. A. Sunyaev and Y. B. Zeldovich, The observations of relic radiation as a test of the nature of $\mathrm{X}$-ray radiation from the clusters of galaxies, Comments Astrophys. Space Phys. 4, 173 (1972). 
[14] J. E. Carlstrom, G. P. Holder, and E. D. Reese, Cosmology with the Sunyaev-Zel'dovich effect, Annu. Rev. Astron. Astrophys. 40, 643 (2002).

[15] T. Kitayama, Cosmological and astrophysical implications of the Sunyaev-Zel'dovich effect, Prog. Theor. Exp. Phys. 2014, 6B111 (2014).

[16] T. Mroczkowski et al., Astrophysics with the spatially and spectrally resolved Sunyaev-Zeldovich effects: A millimetre/ submillimetre probe of the warm and hot Universe, Space Sci. Rev. 215, 17 (2019).

[17] P.-J. Zhang and U.-L. Pen, Deprojecting Sunyaev-Zeldovich statistics, Astrophys. J. 549, 18 (2001).

[18] J. Shao, P. Zhang, W. Lin, and Y. Jing, The thermal SZ tomography, Astrophys. J. 730, 127 (2011).

[19] J. A. Newman, Calibrating redshift distributions beyond spectroscopic limits with cross-correlations, Astrophys. J. 684, 88 (2008).

[20] M. McQuinn and M. White, On using angular crosscorrelations to determine source redshift distributions, Mon. Not. R. Astron. Soc. 433, 2857 (2013).

[21] B. Ménard, R. Scranton, S. Schmidt, C. Morrison, D. Jeong, T. Budavari, and M. Rahman, Clusteringbased redshift estimation: Method and application to data, arXiv:1303.4722.

[22] S. J. Schmidt, B. Ménard, R. Scranton, C. B. Morrison, M. Rahman, and A. M. Hopkins, Inferring the redshift distribution of the cosmic infrared background, Mon. Not. R. Astron. Soc. 446, 2696 (2015).

[23] Y.-K. Chiang and B. Ménard, Extragalactic imprints in galactic dust maps, Astrophys. J. 870, 120 (2019).

[24] Y.-K. Chiang, B. Ménard, and D. Schiminovich, Broadband intensity tomography: Spectral tagging of the cosmic UV background, Astrophys. J. 877, 150 (2019).

[25] V. Vikram, A. Lidz, and B. Jain, A measurement of the galaxy-group thermal Sunyaev-Zel'dovich effect crosscorrelation function, Mon. Not. R. Astron. Soc. 467, 2315 (2017).

[26] R. Makiya, S. Ando, and E. Komatsu, Joint analysis of the thermal Sunyaev-Zeldovich effect and 2MASS galaxies: Probing gas physics in the local Universe and beyond, Mon. Not. R. Astron. Soc. 480, 3928 (2018).

[27] S. Pandey et al. (DES Collaboration), Constraints on the redshift evolution of astrophysical feedback with SunyaevZel'dovich effect cross-correlations, Phys. Rev. D 100, 063519 (2019).

[28] N. Koukoufilippas, D. Alonso, M. Bilicki, and J. A. Peacock, Tomographic measurement of the intergalactic gas pressure through galaxy-tSZ cross-correlations, Mon. Not. R. Astron. Soc. 491, 5464 (2020).

[29] Z. Yan et al., Probing galaxy bias and intergalactic gas pressure with kids Galaxies-tSZ-CMB lensing crosscorrelations, Astron. Astrophys. 651, A76 (2021).

[30] E. Komatsu and T. Kitayama, Sunyaev-Zel'dovich fluctuations from spatial correlations between clusters of galaxies, Astrophys. J. Lett. 526, L1 (1999).

[31] E. Komatsu and U. Seljak, The Sunyaev-Zel'dovich angular power spectrum as a probe of cosmological parameters, Mon. Not. R. Astron. Soc. 336, 1256 (2002).

[32] B. Bolliet, B. Comis, E. Komatsu, and J. F. MacíasPérez, Dark energy constraints from the thermal
Sunyaev-Zeldovich power spectrum, Mon. Not. R. Astron. Soc. 477, 4957 (2018).

[33] R. Makiya, C. Hikage, and E. Komatsu, New constraints on the mass bias of galaxy clusters from the power spectra of the thermal Sunyaev-Zeldovich effect and cosmic shear, Publ. Astron. Soc. Jpn. 72, 26 (2020).

[34] K. Dolag, E. Komatsu, and R. Sunyaev, SZ effects in the Magneticum Pathfinder Simulation: Comparison with the Planck, SPT, and ACT results, Mon. Not. R. Astron. Soc. 463, 1797 (2016).

[35] V. Springel, The cosmological simulation code GADGET-2, Mon. Not. R. Astron. Soc. 364, 1105 (2005).

[36] A. M. Beck et al., An improved SPH scheme for cosmological simulations, Mon. Not. R. Astron. Soc. 455, 2110 (2016).

[37] E. Komatsu et al. (WMAP Collaboration), Seven-year Wilkinson microwave anisotropy probe (WMAP) observations: Cosmological interpretation, Astrophys. J. Suppl. Ser. 192, 18 (2011).

[38] S. Bocquet, A. Saro, K. Dolag, and J. J. Mohr, Halo mass function: Baryon impact, fitting formulae and implications for cluster cosmology, Mon. Not. R. Astron. Soc. 456, 2361 (2016).

[39] N. Gupta, A. Saro, J. Mohr, K. Dolag, and J. Liu, SZE observables, pressure profiles and centre offsets in Magneticum simulation galaxy clusters, Mon. Not. R. Astron. Soc. 469, 3069 (2017).

[40] B. Soergel, A. Saro, T. Giannantonio, G. Efstathiou, and K. Dolag, Cosmology with the pairwise kinematic SZ effect: Calibration and validation using hydrodynamical simulations, Mon. Not. R. Astron. Soc. 478, 5320 (2018).

[41] A. Ragagnin, K. Dolag, L. Moscardini, A. Biviano, and M. D'Onofrio, Dependency of halo concentration on mass, redshift and fossilness in Magneticum hydrodynamic simulations, Mon. Not. R. Astron. Soc. 486, 4001 (2019).

[42] T. Castro, S. Borgani, K. Dolag, V. Marra, M. Quartin, A. Saro, and E. Sefusatti, On the impact of baryons on the halo mass function, bias, and cluster cosmology, Mon. Not. R. Astron. Soc. 500, 2316 (2020).

[43] M. Hirschmann, K. Dolag, A. Saro, L. Bachmann, S. Borgani, and A. Burkert, Cosmological simulations of black hole growth: AGN luminosities and downsizing, Mon. Not. R. Astron. Soc. 442, 2304 (2014).

[44] A. F. Teklu, R.-S. Remus, K. Dolag, A. M. Beck, A. Burkert, A. S. Schmidt, F. Schulze, and L. K. Steinborn, Connecting angular momentum and galactic dynamics: The complex interplay between spin, mass, and morphology, Astrophys. J. 812, 29 (2015).

[45] R.-S. Remus, K. Dolag, and T. L. Hoffmann, The outer halos of very massive galaxies: BCGs and their DSC in the magneticum simulations, Galaxies 5, 49 (2017).

[46] A. F. Teklu, R.-S. Remus, K. Dolag, and A. Burkert, The morphology-density relation: Impact on the satellite fraction, Mon. Not. R. Astron. Soc. 472, 4769 (2017).

[47] R.-S. Remus, K. Dolag, T. Naab, A. Burkert, M. Hirschmann, T. L. Hoffmann, and P. H. Johansson, The co-evolution of total density profiles and central dark matter fractions in simulated early-type galaxies, Mon. Not. R. Astron. Soc. 464, 3742 (2017). 
[48] K. Dolag, E. Mevius, and R.-S. Remus, Distribution and evolution of metals in the magneticum simulations, Galaxies 5, 35 (2017).

[49] L. K. Steinborn, K. Dolag, J. M. Comerford, M. Hirschmann, R.-S. Remus, and A.F. Teklu, Origin and properties of dual and offset active galactic nuclei in a cosmological simulation at $z=2$, Mon. Not. R. Astron. Soc. 458, 1013 (2016).

[50] Planck Collaboration, Planck intermediate results. V. Pressure profiles of galaxy clusters from the SunyaevZeldovich effect, Astron. Astrophys. 550, A131 (2013).

[51] V. Biffi, K. Dolag, and H. Böhringer, Investigating the velocity structure and X-ray observable properties of simulated galaxy clusters with PHOX, Mon. Not. R. Astron. Soc. 428, 1395 (2013).

[52] G. Pollina, N. Hamaus, K. Dolag, J. Weller, M. Baldi, and L. Moscardini, On the linearity of tracer bias around voids, Mon. Not. R. Astron. Soc. 469, 787 (2017).

[53] N. Hand, Y. Feng, F. Beutler, Y. Li, C. Modi, U. Seljak, and Z. Slepian, nbodykit: An open-source, massively parallel toolkit for large-scale structure, Astron. J. 156, 160 (2018).

[54] https://github.com/ryumakiya/pysz.

[55] J. L. Tinker, A. V. Kravtsov, A. Klypin, K. Abazajian, M. S. Warren, G. Yepes, S. Gottlober, and D. E. Holz, Toward a halo mass function for precision cosmology: The limits of universality, Astrophys. J. 688, 709 (2008).

[56] J. L. Tinker, B. E. Robertson, A. V. Kravtsov, A. Klypin, M. S. Warren, G. Yepes, and S. Gottlober, The large scale bias of dark matter halos: Numerical calibration and model tests, Astrophys. J. 724, 878 (2010).

[57] M. Arnaud, G. W. Pratt, R. Piffaretti, H. Boehringer, J. H. Croston, and E. Pointecouteau, The universal galaxy cluster pressure profile from a representative sample of nearby systems (REXCESS) and the Y_SZ-M_500 relation, Astron. Astrophys. 517, A92 (2010).

[58] N. Afshordi, Y.-S. Loh, and M. A. Strauss, Cross-correlation of the cosmic microwave background with the 2MASS galaxy survey: Signatures of dark energy, hot gas, and point sources, Phys. Rev. D 69, 083524 (2004).

[59] R. Voivodic and A. Barreira, Responses of halo occupation distributions: A new ingredient in the halo model \& the impact on galaxy bias, J. Cosmol. Astropart. Phys. 05 (2021) 069.

[60] T. Akahori and K. Yoshikawa, Hydrodynamic simulations of merging galaxy clusters: Non-equilibrium ionization state and two-temperature structure, Publ. Astron. Soc. Jpn. 62, 335 (2010).

[61] C. Avestruz, D. Nagai, E. T. Lau, and K. Nelson, Nonequilibrium electrons in the outskirts of galaxy clusters, Astrophys. J. 808, 176 (2015).

[62] M. Vogelsberger, S. Genel, V. Springel, P. Torrey, D. Sijacki, D. Xu, G. F. Snyder, D. Nelson, and L. Hernquist, Introducing the Illustris Project: Simulating the coevolution of dark and visible matter in the Universe, Mon. Not. R. Astron. Soc. 444, 1518 (2014).

[63] Y. Dubois et al., Dancing in the dark: Galactic properties trace spin swings along the cosmic web, Mon. Not. R. Astron. Soc. 444, 1453 (2014).

[64] A. M. C. L. Brun, I. G. McCarthy, J. Schaye, and T. J. Ponman, Towards a realistic population of simulated galaxy groups and clusters, Mon. Not. R. Astron. Soc. 441, 1270 (2014).

[65] J. Schaye et al., The EAGLE project: Simulating the evolution and assembly of galaxies and their environments, Mon. Not. R. Astron. Soc. 446, 521 (2015).

[66] I. G. McCarthy, J. Schaye, S. Bird, and A. M. C. Le Brun, The BAHAMAS project: Calibrated hydrodynamical simulations for large-scale structure cosmology, Mon. Not. R. Astron. Soc. 465, 2936 (2017).

[67] A. Pillepich et al., Simulating galaxy formation with the illustristng model, Mon. Not. R. Astron. Soc. 473, 4077 (2018). 